\title{
Changes in biomarkers of endothelial function in the blood after intracerebral hemorrhage in rats with type 2 diabetes mellitus
}

\author{
A. E. Lievykh*, V. A. Tkachenko*, Y. V. Kharchenko*, A. I. Shevtsova****, G. A. Ushakova**, V. I. Zhyliuk* \\ *Dnipro State Medical University, Dnipro, Ukraine \\ **Oles Honchar Dnipro National University, Dnipro, Ukraine
}

Article info

Received 20.10.2021

Received in revised form 11.11 .2021

Accepted 14.11.2021

Dnipro State Medical University, Vernadsky st., 9 Dnipro, 49044, Ukraine. Tel.: + 38-050-565-88-23. E-mail:vzhyliuk@gmail.com

Oles Honchar Dnipro National University, Gaharin av., 72 Dipro, 49010, Ukraine. $\mathrm{Tel} \cdot$ + 38-067-632-36-13. E-mail: ushakova_g@ukrnet

Lievykh, A. E., Tkachenko, V. A., Kharchenko, Y. V., Shevtsova, A. I., Ushakova, G. A., \& Zhyliuk, V. I. (2021). Changes in biomarkers of endothelial function in the blood after intracerebral hemorrhage in rats with type 2 diabetes mellitus. Regulatory Mechanisms in Biosystems, 12(4), 733-739. doi:10.15421/0221101

Clinical evidence suggests that type 2 diabetes mellitus can increase the risk of intracerebral hemorrhage and provocation of neurodegeneration. This study was aimed at evaluating biomarkers of glycemic control, lipid profile, oxidative modification of proteins, as well as the functional state of endothelium in Wistar rats with type 2 diabetes mellitus complicated by intracerebral hemorrhage. Experimental type 2 diabetes mellitus was induced by intraperitoneal injection of streptozotocin $(65 \mathrm{mg} / \mathrm{kg})$ and nicotinamide $(230 \mathrm{mg} / \mathrm{kg})$. The intracerebral hemorrhage was induced by microinjection of sterile saline containing $0.2 \mathrm{IU}$ bacterial collagenase into the striatum. Assessed biomarkers included the area under glycemic curve, glycated hemoglobin, total cholesterol, triglyceride, high-density lipoprotein, advanced glycation end products, markers of oxidative modification of proteins - aldehyde- and ketonephenylhydrazones, and markers of endothelial dysfunction - homocysteine, endothelin-1, von Willebrand factor and asymmetric dimethylarginine in blood serum. Both rats with type 2 diabetes mellitus and rats with intracerebral hemorrhage and diabetes had a significant elevated glycemic control as compared to intact animals. But combined pathology was additionally characterized by an impairment of lipid profile (increased triglyceride level and decreased as total cholesterol and high-density lipoprotein) resulting in a rise in the atherogenic index of plasma. A significant increase in the content of the markers of oxidative modification of proteins was observed in both experimental groups. But the rats with intracerebral hemorrhage and diabetes only had higher levels of advanced glycation end products in comparison with intact animals. The highest levels of endothelin-1, as a biomarker of endothelial dysfunction, were observed in animals with intracerebral hemorrhage and diabetes. Homocysteine and von Willebrand factor were elevated in rats with type 2 diabetes mellitus, while acute intracerebral hemorrhage did not potentiate the further growth in its levels. Such effect was not accompanied by a marked increase of asymmetric dimethylarginine level in blood serum, although there was a clear trend. In conclusion, the development of intracerebral hemorrhage in rats with type 2 diabetes mellitus can intensify the manifestations of oxidative stress, worsen lipid profile, and aggravate endothelial dysfunction. In this case, the pathological process may have the character of a "vicious circle".

Keywords: glycemic control; lipid profile; endothelin-1; von Willebrand factor; oxidative modification of proteins; advanced glycation end products.

\section{Introduction}

Diabetes mellitus (DM) is one of the fastest growing global health challenges of the 21st century. Based on the International Diabetes Federation Diabetes Atlas (2019), 463 million adults aged 20-79 years (prevalence of $9.6 \%$ ) are estimated to be living with diabetes mellitus today, and 700 million (prevalence of $10.9 \%$ ) are projected to be living with the condition by 2045. It is well established that type 2 diabetes mellitus (T2DM) is associated to a variety of non-modifiable and modifiable risk factors. Type 2 diabetes mellitus belongs to a group of chronic metabolic diseases that is caused by insulin resistance or deficiency, resulting in increased blood glucose levels. This form of diabetes is the most common, as it accounts for $90-95 \%$ of patients with diabetes mellitus. Poorly controlled T2DM is associated with increased morbidity and mortality, and the leading cause of death is cardiovascular disease, including atherosclerosis and hypertension (Su, 2015; Mashayekhi-Sardoo et al., 2021; Strain \& Paldánius, 2021). Insulin resistance in type 2 diabetes mellitus is associated with many risk factors that usually precede the development of hyperglycemia. These typically include obesity, dyslipidemia, which is characterized by high triglycerides, elevated blood pressure, oxidative stress, and endothelial dysfunction (ED) (Su, 2015). Endothelial cells, as well as their main products - nitric oxide (NO), prostacyclin, endothelin-1
(ET-1), angiotensin II, von Willebrand factor (vWF), tissue plasminogen activator and reactive oxygen species (ROS) - play a key role in the regulation of vascular homeostasis. Diabetes-induced endothelial dysfunction is a critical and initiating factor in the development of diabetic vascular complications. It is characterized by reduced synthesis and bioavailability of NO, reduced synthesis of prostacyclin and endothelium-derived hyperpolarizing factor (EDHF) and increased production or action of endothelial vasoconstrictors (ET-1, vWF, etc.) (Shi \& Vanhoutte, 2017). Asymmetric dimethylarginine (ADMA) is a competitive inhibitor of endothelial nitric oxide synthase (NOS) and has recently been considered as one of the key biomarkers of endothelial dysfunction and inducers of atherogenesis, as well as a prognostic marker of cardiovascular events and mortality (Anderssohn et al., 2010; Avci et al., 2020). A positive association between ADMA and insulin resistance has also been identified recently (El Assar et al., 2016; Lee et al., 2018).

At the same time, changes in the blood plasma lipid profile are also major risk factors for cardiovascular events. Dyslipidemia in the form of increased levels of low-density lipoprotein (LDL) cholesterol, total cholesterol (TC), triglycerides (TG), and decreased levels of high-density lipoprotein (HDL) cholesterol is the cause of atherosclerosis. Nowadays LDL is considered the main therapeutic target. However, even with complete recovery of $\mathrm{LDL}$ levels, the risk of cardiovascular events remains high $(\mathrm{Li}$ 
et al., 2021). In addition, hypertension and dyslipidemia lead to an imbalance in angiotensin II - bradykinin homeostasis, which is an additional factor in the development of ED and changes in blood vessels that contribute to atherosclerosis and thrombosis (Lancellotti et al., 2018).

It is assumed that the greatest role in reducing endothelium-dependent vasodilation belongs to the development of intracellular oxidative stress. Free radical oxidation significantly reduces endothelial production of NO, which is rapidly degraded by superoxide and peroxide-anions with the formation of an extremely reactogenic compound, peroxynitrite, that is converted into unstable peroxynitric acid, which forms a hydroxyl radical (O-) (Anderssohn et al., 2010). Oxidative modification of proteins (OMP) is one of the early and the most reliable markers of tissue damage in many pathological conditions that involve free radicals, including T2DM. Different tissue proteins but not lipids or nucleic acids are effective traps of the generated ROS that are overproduced or accumulated in oxidative stress (Butko et al., 2013).

In addition, the higher levels of glucose and/or lipids in diabetes are responsible for the increased production of highly reactive carbonyl compounds - a condition referred to as "carbonyl stress". In particular, glycotoxins and lipotoxins are able to react rapidly and damage various cellular and extracellular molecules forming advanced glycation end products (AGEs) (Nafisa et al., 2018; Yaribeygi et al., 2020; Menini et al., 2021). Binding of AGEs to their receptors (RAGE) increases the intracellular enzymatic production of superoxide, which in turn not only has direct harmful effects, but also activates various intracellular signaling pathways, such as NF- $\mathrm{kB}$, p38 MAPK, JNK / SAPK, hexosamine biosynthesis pathway, protein kinase C, AGEs / RAGE, TNF $\alpha$ and sorbitol synthesis, which further enhances the production of superoxide and creates a "vicious circle effect". In addition, advanced glycation end products can accumulate in the vascular wall thereby reducing the activity of $\mathrm{NO}$ and the expression of endothelial nitric oxide synthase (eNOS), as well as increasing the expression of ET-1, which underlie the development of endothelial dysfunction (Ren et al., 2017; Nafisa et al., 2018). Nowadays protein carbonylation is one of the most harmful and irreversible of its modifications and is considered a key factor in the progression of diabetes and associated complications (Nair et al., 2021).

Hyperhomocysteinemia is also an important factor in endothelial dysfunction as it may reduce the synthesis and bioavailability of NO and EDHF, promote the production of vasoconstrictor prostanoids and activation of angiotensin II type 1 (AT1) receptors, as well as ROS generation due to phosphorylation of NADPH oxidase or increasing the activity of angiotensin-converting enzyme (ACE) and the formation of angiotensin II, which also activates NADPH oxidase (Su, 2015).

Intracerebral hemorrhage (ICH) is the most common cause of traumatic brain injury and the second most common cause of stroke as it occurs spontaneously in hemorrhagic stroke. Non-traumatic intracerebral hemorrhage leads to spontaneous bleeding into brain tissue and causes high disability and mortality (Yu et al., 2015). There are many risk factors associated with non-traumatic ICH, but hyperglycemia is one of the most important causes of its occurrence (Hill, 2014). Although diabetes is an independent risk factor primarily for ischemic stroke, it may also be associated with an increased risk of intracerebral hemorrhage, which is directly associated with diabetes duration, and such patients have more severe complications after ICH (Yu et al., 2015). The prevalence of diabetes was higher in people with ischemic stroke (33\%, 95\% CI 28-38\%) compared with hemorrhagic stroke (26\%, 95\% CI 19-33\%) and in the studies that included both stroke types (24\%, 95\% CI 20-28\%) (Lau et al., 2018). A possible J-shaped relationship has been established between intracerebral hemorrhage and glycated hemoglobin $(\mathrm{HbAlc})$ levels, suggesting that both poor control and extremely intensive control of diabetes may carry an increased risk of ICH (Saliba et al., 2019). However, the mechanisms responsible for the association between intracerebral hemorrhage and diabetes mellitus have not been sufficiently studied yet.

Based on the prominent changes in carbohydrate and lipid metabolism in rats with diabetes mellitus, the primary purpose of this study was investigation of evaluating biomarkers of glycemic and oxidation control as well as functional state of endothelium of rats with type 2 diabetes mellitus complicated by intracerebral hemorrhage.

\section{Materials and methods}

The study design, all experimental protocols and euthanasia of animals were approved by the Biomedical Ethics Committee of the Dnipro State Medical University (protocol No. 8 dated 17.12.2019). Experiments were performed in compliance with the Directive 86/609/EEC on the Protection of Animals Used for Experimental and Other Scientific Purposes and the order No. 3447-IV of 21.02.2006 new editorship of 08.08.2021 "On Protection of Animals against Abuse" and the Provisions of the "European Convention for the Protection of Animals used for Experimental and other Scientific Purposes" (Ukraine).

The study was carried out on 24 male Wistar rats weighing 200$250 \mathrm{~g}$. Type 2 diabetes mellitus was simulated with a single intraperitoneal injection of nicotinamide (NA, $230 \mathrm{mg} / \mathrm{kg}$ ) and streptozotocin (STZ, $65 \mathrm{mg} / \mathrm{kg}$ ) in citrate buffer $(\mathrm{pH}=4.5,0.1 \mathrm{M})$ to overnight fasted rats (Ghasemi et al., 2014; Potârniche et al., 2018). Blood glucose level was measured 72 hours after NA/STZ injection. Animals with values less than $8.3 \mathrm{mmol} / \mathrm{L}$ were excluded from the study (Potârniche et al., 2018). Intracerebral hemorrhage (ICH) in rats was induced by microinjection of $1 \mu \mathrm{L}$ of bacterial collagenase $0.2 \mathrm{IU} / \mu \mathrm{L}$ (Type IV-S) (Chen et al., 2019). On the 60th day after NA/STZ injection, a Hamilton microsyringe was inserted into the area of the striatum of anesthetized rats by the following stereotactic coordinates: $0.2 \mathrm{~mm}$ anterior, $2.8-3.0 \mathrm{~mm}$ lateral, and $5.5 \mathrm{~mm}$ ventral to the bregma.

According to the result of the oral glucose tolerance test, all rats with a similar degree of glycemia were randomly divided into three groups: group 1 - control $(n=8)$; group 2 - type 2 diabetes mellitus $(n=9)$; group 3 -intracerebral hemorrhage under type 2 diabetes mellitus $(n=7)$.

Blood glucose level was measured with the blood glucose meter Bionime Rightest GM300 (Bionime Corporation, Switzerland) in blood samples obtained from the tail vein. The oral glucose tolerance test was performed on the 69th day of the study. Overnight fasted animals were given $2 \mathrm{~g} / \mathrm{kg}$ of $20 \%$ glucose solution by intragastric gavage 2 hours after drug administration. The area under the glycemic curve (AUC) was calculated using GraphPad Prism 9.0 software and expressed as $\mathrm{mmol} / \mathrm{L} \times 120 \mathrm{~min}$.

On the 70th day of the study, blood samples were obtained by intracardiac puncture from the right ventricle of the heart of anesthetized rats.

Glycated hemoglobin ( $\mathrm{HbAlc}$ ) was measured spectrophotometrically in whole blood samples using a standard HbAlc kit ("Reagent", Ukraine). The principle of the method is based on the presence of 1-deoxy-1(N-valyl)fructose in a stable form of HbA1C. Further, it is dehydrated by phosphoric acid to 5-hydroxymethyl-2-furaldehyde that forms a colour complex with 2-thiobarbituric acid with the maximum absorption at $443 \mathrm{~nm}$ (Gabbay et al., 1979). The content of HbAlc was expressed as $\mu \mathrm{mol}$ fructose/g $\mathrm{Hb}$.

Total cholesterol, triglyceride, high-density lipoprotein, and glucose levels were measured in blood serum using diagnostic kits ("Reagent"; Ukraine). The content of TC, TG, HDL, and glucose was expressed as $\mathrm{mmol} / \mathrm{L}$. Atherogenic indices such as TG/HDL ratio and atherogenic index of plasma (AIP) were calculated by using the values of lipid profile parameters (Ho et al., 2015; Çoban et al., 2018). AIP was calculated using the following formula AIP $=\log (\mathrm{TG} / \mathrm{HDL}$ ) (Frohlich \& Dobiásová, 2003; Zhu et al., 2018).

The level of advanced glycated end products (AGEs) was measured by fluorescence method (Münch, 1997), using Hoefer DQ 2000 Fluorometer (USA) with fixed wavelengths (excitation $/$ emission $=365 \mathrm{~nm} /$ $460 \mathrm{~nm}$ ). The results were expressed as arbitrary units (AU) per mg of protein.

The markers of oxidative modification of proteins (aldehydephenylhydrazones and ketonephenylhydrazones) were evaluated spectrophotometrically by the method of B. Halliwell which is based on the reaction of interaction of oxidized amino-acid residues with 2,4-dinitro-phenylhydrazine $(2,4-\mathrm{DNPhH})$ with formation of 2,4-dinitro-phenylhydrazones, which are proportional to the optical density at 270 and $363 \mathrm{~nm}$ (Butko et al., 2013). The content of carbonyl derivatives of oxidized proteins was expressed in units of optical density per mg of protein.

Homocysteine level in serum was measured by enzymatic method using "Homocysteine, enzymatic cycling" kit ("DIALAB ${ }^{\circledR}$ G.m.b.H." 
Wr. Neudorf, Austria). This marker was assessed with a semiautomatic biochemical analyzer HTI BioChem SA (High Technology Inc., USA).

The level of endothelin-1, von Willebrand factor, asymmetric dimethylarginine in blood serum were assessed using "Rat Endothelin-1 (ET-1) ELISA Kit, Catalog \# MBS3808173", "Rat Von Willebrand Factor (vWF) ELISA Kit, Catalog \# MBS 775527” (MyBioSourse, Inc., San Diego, CA, USA), and "ADMA (Asymmetrical Dimethylarginine) ELISA Kit, Catalog \# E-EL-0042" (ElabscienceBiotechnology Inc., Houston, TX, USA), correspondingly according to the manufacturer protocol. Absorbance was read on an ELISA microplate reader RT 2100 (Rayto, China) at $450 \mathrm{~nm}$.

Statistical data analysis was performed by GraphPad Prism 9.0 (GraphPad Software, Inc., La Jolla, CA, USA, 2020). All results are expressed as $\mathrm{x} \pm \mathrm{SD}$ (standard deviation). Statistical significance $(\mathrm{P}<0.05)$ was determined by one-way ANOVA for normally distributed variables; and Kruskal-Wallis H-test for non-normally distributed variables with Bonferroni correction.

\section{Results}

The results of the study showed that the development of type 2 diabetes mellitus in rats led to a significant increase in basal glycemia, but intracerebral hemorrhage did not affect its severity (Fig. 1a).

The changes in blood glucose AUC in all groups of rats on the $69^{\text {th }}$ day of the experiment were shown (Fig. 1a, b). According to the data obtained, the course of type 2 diabetes mellitus (group 2) led to the development of glucose tolerance evidenced by an increase in glycemic AUC by $58.1 \%(\mathrm{P}=0.0118)$. Meanwhile, modeling intracerebral hemorrhage under diabetes (group 3) did not affect glucose tolerance in this test significantly, and AUC value was higher by $75.3 \%(\mathrm{P}=0.0007)$ as compared to the negative control group (group 1). Additionally, it was found that NA/STZ-treated rats had increased level of glycated hemoglobin HbAlc by $37.3 \%(\mathrm{P}=0.0187)$ compared to the animals in the control group. However, this marker was higher by $53.9 \%(\mathrm{P}=0.0015)$ in rats with $\mathrm{T} 2 \mathrm{DM}+\mathrm{ICH}$ as compared to the control group (Fig. 1c).
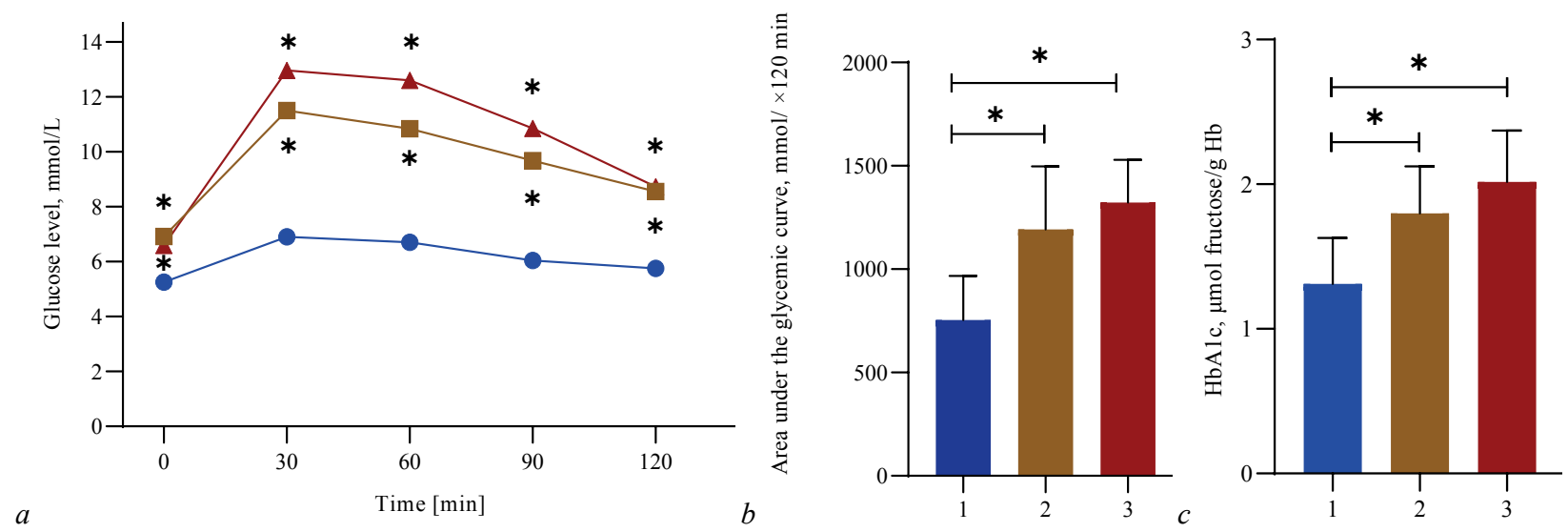

Fig. 1. Markers of glycemic control in rats with type 2 diabetes mellitus and intracerebral hemorrhage:

all data are presented as $\mathrm{x} \pm \mathrm{SD} ; 1$ - control rats $(\mathrm{n}=8) ; 2$ - rats with type 2 diabetes mellitus $(\mathrm{n}=9) ; 3$-rats with intracerebral hemorrhage under type 2 diabetes mellitus ( $(n=7) ; a$-concentration of glucose in the blood serum due to oral glucose tolerance test (on the 69th day of the experiment); $b$ - the area under the glycemic curve (on the 69th day of the experiment); $c$ - concentration of glycated hemoglobin (on the 70th day of the experiment); ${ }^{*}-\mathrm{P}<0.05$ with Bonferroni correction (in Fig. 1a compared to group 1)

According to the data in Figure 2, the diabetic animals were characterized by certain changes in lipid profile. In this regard, it is noteworthy that there was a moderate lowering in total cholesterol (TC) levels in rats with T2DM, which progressed under conditions of acute brain hemorrhage, and TC was decreased by $22.2 \%(\mathrm{P}=0.0309)$ in comparison with the control group (Fig. 2a). Moreover, HDL cholesterol level (Fig. 1b) in group 3 was less by $32.9 \%(\mathrm{P}=0.0243)$ and TG level (Fig. 1c) was higher by $31.4 \%(P=0.0305)$ than the values of naive animals (group 1$)$. Simultaneously, the ratio of AIP (Fig. 2d) was 2.97 times higher $(\mathrm{P}=0.0127)$ than the values of group 1. Experimental diabetes was characterized by a non-significant increase of advanced glycated end products, but acute intracerebral hemorrhage in rats with T2DM led to a statistically significant rise in the AGEs level by $53.9 \%(\mathrm{P}=0.0409)$ in comparison with control group 1 (Fig. 3a). At the same time, there was a significant increase in the content of the markers of oxidative modification of proteins (APH and $\mathrm{KPH})$ in both groups 2 (by $11.8 \%(\mathrm{P}=0.01)$ and $34.0 \%(\mathrm{P}=$ $0.0099)$, respectively) and group 3 (by $10.5 \%(\mathrm{P}=0.0345)$ and $38.7 \%$ $(\mathrm{P}=0.0009$ ), respectively) compared to the control group (Fig. 3b, c).
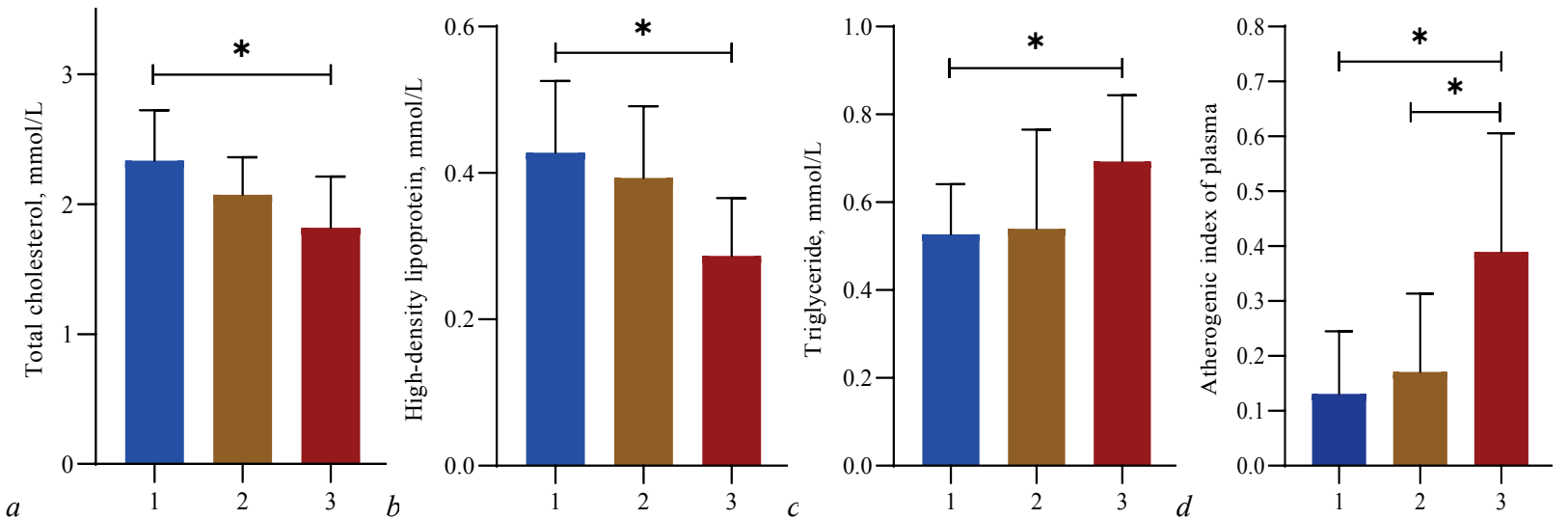

Fig. 2. Markers of lipid profile in the blood serum of rats with type 2 diabetes mellitus and intracerebral hemorrhage on the 70th day of the experiment: abrevation for groups see Figure 1; $a$-concentration of cholesterol; $b$-concentration of triglyceride; $c$ - concentration of high-density lipoprotein; $d$-atherogenic index of plasma; ${ }^{*}-\mathrm{P}<0.05$ with Bonferroni correction 

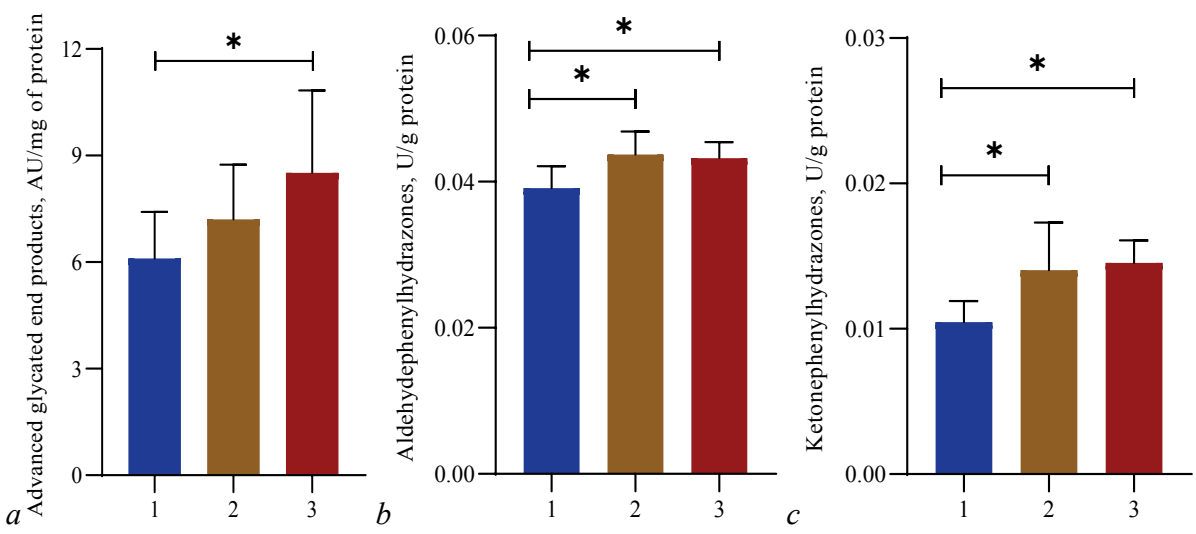

Fig. 3. The content of advanced glycated end products $(a)$ and products of oxidizing modification of proteins (APH - aldehydephenylhydrazones) $(b)$ and (KPH - ketonephenylhydrazones) $(c)$ in the blood serum of rats with type 2 diabetes mellitus and intracerebral hemorrhage on the 70th day of the experiment: abrevation for groups see Figure $1 ;{ }^{*}-\mathrm{P}<0.05$ with Bonferroni correction

Homocysteine, as a predictor of endothelial dysfunction and atherogenesis, also showed a clear tendency to increase in rats with T2DM, while acute intracerebral hemorrhage does not potentiate the further increase in its level in these conditions (Fig. 4a).

The highest level of endothelin- 1 in the blood serum was observed in animals of group 3. It was increased by $5.1 \%(\mathrm{P}=0.0437)$ as compared to the level in group 1 (Fig. 4b). Similar changes were observed also for von
Willebrand factor level (Fig. 4c). It was found that both type 2 diabetes mellitus and T2DM complicated by intracerebral hemorrhage were characterized by an increased serum vWF level (by $10.4 \%, \mathrm{P}=0.0177$ and $15.2 \%, \mathrm{P}=0.0017$, respectively).

It should be noted that changes of asymmetrical dimethylarginine level were present in the form of an upward trend only in both groups 3 and 3 without any statistical significance (Fig. 4d).
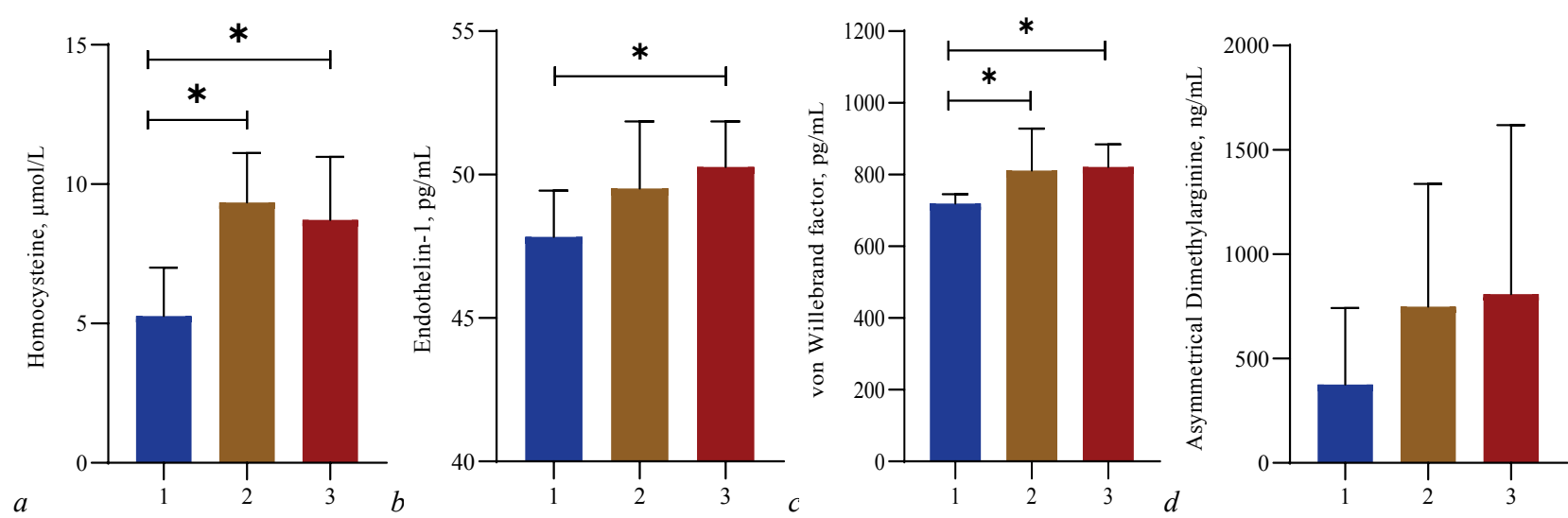

Fig. 4. Markers of endothelial function in the blood serum of rats with type 2 diabetes mellitus and intracerebral hemorrhage on the 70th day of the experiment: abbrevation for groups see Fig. 1; concentration of homocysteine (a), endothelin-1 (b), von Willebrand factor (c), asymmetrical dimethylarginine (d); ${ }^{*}-\mathrm{P}<0.05$ with Bonferroni correction

\section{Discussion}

The present study aimed to determine the existence of the influence of acute intracerebral hemorrhage under type 2 diabetes mellitus and the manifestations of endothelial dysfunction caused by this pathological condition. It was found that although there were no statistically significant differences between the groups of positive control, the values of markers representing the course of diabetes mellitus and its manifestations (glucose tolerance) were higher in the group of animals with intracerebral hemorrhage. The obtained data agree with the results of clinical studies conducted earlier. It is known that the development of acute hemorrhagic stroke can provoke "stress" hyperglycemia, even in patients without diabetes or with its latent form, which may be associated with early patient mortality and more severe consequences of stroke (Lau et al., 2019). Higher glucose levels were shown to be accompanied by a higher inhospital mortality rate of patients with hemorrhagic stroke compared to patients with normal glycemic levels (Snarska et al., 2017). Another study also found that every increase in $1.0 \mathrm{mmol} / \mathrm{L}$ in blood glucose over $5.0 \mathrm{mmol} / \mathrm{L}$ was associated with a $33 \%$ increased probability of 30-day mortality in patients with hemorrhagic stroke, after adjusting for diabetic status (Godoy et al., 2008).

Dyslipidemia in diabetes is characterized by the presence of potentially atherogenic lipids, including high level of triglyceride in the blood, moderately elevated LDL contents, and low concentration of HDL (Zambon, 2020). Atherosclerosis is the key factor leading to vascular complications in patients with type 2 diabetes mellitus. Moreover, a lot of clinical trials report the importance of endothelial dysfunction and platelet hyperactivity in the pathogenesis of atherosclerotic vascular complications by diabetes mellitus (Kaur et al., 2018). In our study, statistically significant changes in lipid profile were found only in the group of diabetic animals with intracerebral hemorrhage, which to some extent may indicate a deterioration in lipid metabolism caused by acute cerebral hemorrhage in T2DM. At the same time, a progressive decrease in total cholesterol and HDL levels is also notable, as well as increase of triglyceride concentration in the blood of rats in the groups 2 and 3 compared with intact animals. The ratio of triglycerides to high-density lipoprotein (TG/HDL) is now considered as a biomarker of insulin resistance and atherogenicity. And the change of this marker could be an additional confirmation of type 2 diabetes mellitus and atherogenesis in our study (Ho et al., 2015; Çoban, 2018). Elevated TG levels reduce both the number and activity of insulin receptors on adipocytes. In addition, it is known that triglycerides are competitors of glucose for entry into the cell, and hypertriglyceridemia leads to the disruption of glucose oxidation. As a result, hypertriglyceridemia can cause glucose and insulin tolerance. In turn, low HDL levels also negatively affect the function of $\beta$-cells both by reducing their sensitivity to glucose and by suppressing insulin secretion. However, increased 
levels of TG and free fatty acids along with a decrease in HDL levels can also be a consequence of insulin resistance, and the above phenomena can indicate the presence of a "vicious circle" (Li et al., 2021). On the other hand, it is known that increased levels of triglycerides promote atherogenesis under the arterial intima, as well as inhibiting fibrinolytic function and activating the extrinsic blood coagulation system, which leads to an increased risk of stroke. Hypertriglyceridemia can also be identified as a risk factor for cerebral hemorrhage. In addition, it is known that the HDL levels in patients with intracerebral hemorrhage are much lower, and the degree of HDL reduction is associated with the severity of hemorrhage (Wang et al., 2018). Due to some inconsistencies in the available literature, which are associated with the reduction of total cholesterol in the blood in different pathological conditions, the advantage of using TC as a marker of atherogenesis becomes somewhat questionable, and the data obtained in the present study are the subject of further research. In recent decades, epidemiologists and clinicians have assessed and predicted the risks of cardiovascular diseases associated with atherogenesis mainly by LDL levels. However, numerous studies indicate the importance not of the actual values of individual parameters of the lipid profile, but the ratio of lipids, which can be important markers of cardiovascular risk due to the imbalance between atherogenic and antiatherogenic lipoproteins. The atherogenic index of plasma (AIP) is the most prognostic and sensitive marker compared to the other three atherogenesis markers, such as Castelli risk index-I (TC/HDL), Castelli risk index-II (LDL/HDL), and atherogenic factor ((TC-HDL)/HDL). An isolated increase in TG levels, although it increases the risk of coronary heart disease, can be balanced by HDL, which has an antiatherogenic effect (Bo et al., 2018). A marked increase of AIP in diabetic animals with intracerebral hemorrhage is an evidence of intensification of changes in lipid metabolism under type 2 diabetes mellitus, which may contribute to active atherogenesis and a significant increase in the risk of cardiovascular complications under these conditions and require urgent medical correction. Although the relationship between lipid levels, lipid-lowering therapy, and the risk of intracerebral hemorrhage remains unclear, our findings are consistent with previous research (Ma et al., 2016). According to these data, low serum levels of TC and HDL may be associated with an increased risk of intracerebral hemorrhage, including during therapy with high doses of statins. However, the causes of dyslipidemia (particularly, decrease of TC levels) in the conditions of type 2 diabetes mellitus (both isolated and complicated by intracerebral hemorrhage) require further studies.

Hyperglycemia is thought to trigger vascular damage by creating imbalance between NO bioavailability and accumulation of ROS. Furthermore, hyperglycemia damages the vascular bed by several cellular mechanisms, comprising of enhanced production of intracellular advanced glycation end products (AGEs), increased expression of AGE receptors (RAGE) and ligands, augmented polyol and hexosamine flux, activation of protein kinase $\mathrm{C}$ (PKC) and overactivation of the hexosamine pathway. AGEs formation further lead to alteration of structural, functional and receptor recognition properties of matrix components. Sequentially, binding of AGEs to their receptors increases superoxide production, which promotes macrophage induced vascular inflammation. Furthermore, AGEs also induce decreased endothelial $\mathrm{NO}$ synthase (eNOS) expression as well as NO synthesis and increased endothelin-1 expression, leading to endothelial dysfunction (Odegaard et al., 2016; Kaur et al., 2018). According to our data, the course of T2DM (both isolated and complicated by ICH) was characterized by a significant intensification of oxidative modification processes and increasing content of early and late markers of protein carbonylation. Simultaneously, it should be noted that AGEs levels tended to increase in both experimental groups, but statistically significant differences were found only in the group of animals with T2DM+ICH. Thus, under hyperglycemic conditions, altered formation of various biochemicals including AGEs and ROS, significantly contributes to development of complications in diabetes mellitus, and the occurrence of intracerebral hemorrhage contributes to the severity of these processes.

Vascular endothelium is a major target of oxidative stress, playing a critical role in the pathophysiology of several vascular diseases and disorders, and its alteration significantly contributes to diabetic vascular pathology. It is well known that hyperglycemia is able to cause endothelial dysfunction: most observations suggest that the damage from hyperglyc- emia to endothelium is secondary to oxidative stress, but the data available in the literature seem to highlight more mechanisms that are heterogeneous and too complex to explain endothelial damage from hyperglycemia. It is important to underline that ED is related to specific pathophysiological mechanisms that involve: alterations of the substrate/enzyme ratio; alterations in the expression/structure of NOS; signal alterations; and alterations of the availability of cofactors and destruction of $\mathrm{NO}$ (Carrizzo et al., 2018). Endothelial dysfunction is the key event that initiates the inflammatory mechanisms associated with vascular complications in T2DM patients (Dhananjayan et al., 2016). Furthermore, it is an initial event of atherogenesis, which involves imbalance in the tightly regulated equilibrium of vasodilators and vasoconstrictors together with the inhibition of the anticoagulant system.

Homocysteine (Hcy) is considered a risk factor for atherosclerosis and cardiovascular disease (CVD), but the molecular basis of these associations remains elusive. The impairment of endothelial function, a key initial event in the setting of atherosclerosis and CVD, is recurrently observed in hyperhomocysteinemia. Various observations may explain the vascular toxicity associated with hyperhomocysteinemia. For instance, Hcy interferes with the production of nitric oxide (NO), a gaseous master regulator of endothelial homeostasis. Moreover, Hcy deregulates the signaling pathways associated with another essential endothelial gasotransmitter: hydrogen sulfide (Esse et al., 2019). Endothelial oxidative stress because of ROS accumulation is the major mechanism that mediates homocysteine-induced vascular injury. In addition to the interaction of homocysteine with cysteine to form a disulphide bond, homocysteine also directly inhibits the activity of antioxidants, thereby disrupting SOD, activating NADPH oxidases (NOXs) and subsequently producing superoxide anion, causing an accumulation of ROS. The generated ROS further activate the transcriptional activity of NF- $\mathrm{kB}$, which results in the expression of proinflammatory genes and vascular inflammation. ROS disturb lipoprotein metabolism, contributing to the growth of atherosclerotic vascular lesions (Škovierová et al., 2016; Fu et al., 2018; Esse et al., 2019). The data obtained in the present study indicate an increase in blood Hcy levels in rats with experimental T2DM and are consistent with previous studies (Elias et al., 2005). Hyperhomocysteinemia under these conditions can be aggravating and intensify the development of endothelial dysfunction and atherogenesis. However, modeling of ICH did not lead to a further increase in the levels of this marker in the blood. It has been suggested that negative endotheliotropic effects of Hcy may be mediated by asymmetrical dimethylarginine (Esse et al., 2019).

$\mathrm{NO}$ is a key vasodilator endothelial factor formed by the oxidation of arginine by nitric oxide synthase (NOS). Moreover, this reaction requires the presence of NADPH and $\mathrm{O}_{2}$, and its result is the formation of NO and citrulline as end products. NOS activity is known to be inhibited by methylated arginine analogs, such as NG-Methyl-L-arginine (L-NMMA) and asymmetrical dimethylarginine, which are synthesized in vivo by arginine methyltransferases (Leiper \& Vallance, 1999). Lysis of proteins containing L-NMMA and ADMA promotes their transport into the cytosol and further from the cell into the blood. It is known that increased level of circulating asymmetrical dimethylarginine is associated with atherogenesis and endothelial dysfunction (Anderssohn et al., 2010; Esse et al., 2019; Avci et al., 2020). However, the association between asymmetrical dimethylarginine and diabetes remains questionable to this day. It probably depends on both the type and stage of diabetes. Contradictory data on the cardiovascular risk in people with and without diabetes mellitus have been reported, and the association of ADMA with diabetes remains controversial, possibly as to the type and stage of diabetes. Clinical and experimental data suggest a multifaceted relationship between ADMA and insulin metabolism and its effects, on the one hand, and the use of ADMA and glucose, on the other hand (Anderssohn et al., 2010). This can be confirmed by the results of the present study as a statistically significant increase in Hcy levels in the blood of rats with T2DM (both isolated and complicated by ICH). Such effect was not accompanied by a marked increase in ADMA levels, although there was a clear trend.

The development of endothelial dysfunction is further confirmed in the present study by increasing levels of endothelin-1. Moreover, modeling of ICH contributed to higher levels of this key vasoconstrictor. According to the literature, hyperglycemia causes overproduction of ET-1. 
High glucose-induced ET-1 production in endothelial cells and ET-1-mediated endothelial cell inflammation can be mediated by adapter molecule TRAF3 interacting protein 2 (TRAF3IP2). Previous studies identified endothelial TRAF3IP2 as a potential new therapeutic target to suppress ET-1 production and associated vascular complications in diabetes (Padilla et al., 2018). It is also known that there is a positive correlation between plasma ET-1 levels and microangiopathy in patients with type 2 diabetes mellitus. In addition, ET-1 can cause a decrease in tissue sensitivity to insulin and may be involved in the development of metabolic syndrome. The vasoconstrictive effect of ET-1 may be enhanced by its inhibitory effect on NO production (Kalani et al., 2008). A correlation between ET-1 levels and ICH development has also been identified (Wang et al., 2018).

In experimental models, von Willebrand factor supports the activation of multiple inflammatory pathways, such as complement cascade and NETosis, promotes atherosclerosis favouring plaque progression and complication, and exacerbates ischemia/reperfusion injury (Gragnano et al., 2017). The elevation of plasma vWF levels in the course of diabetes mellitus may indicate both the presence of endothelial dysfunction or vascular inflammation, and a significant risk factor for thrombosis (Shahidi Anderssohn et al., 2010). An increase in plasma vWF levels can be observed in traumatic brain injury and be an unfavourable prognostic marker of this pathological process (De Oliveira et al., 2007; Hubbard et al., 2021). Moreover, vWF levels in cases of focal brain injury were significantly higher than in cases of diffuse axonal lesions. In addition, patients with delayed traumatic intracerebral hemorrhage had significantly higher vWF levels as compared to patients without ICH (Yokota, 2007). Although in the present study intracerebral hemorrhage did not lead to a further increase in vWF levels in diabetic rats, vWF may adversely affect the course of this pathology.

\section{Conclusion}

Development of intracerebral hemorrhage on the background of diabetes mellitus is aggravating in relation to lipid and carbohydrate metabolism changes, as well as the functional state of the vascular wall. Concurrently, diabetes-induced endothelial dysfunction is also likely to exacerbate disorders caused by the development of intracerebral hemorrhage. In this case, the pathological process may have the character of a "vicious circle". Joint assessment of glycemic and atherogenic indices together with biomarkers of glycation end products and oxidized proteins in the blood in relation to the assessment of vascular endothelial status provides a comprehensive picture of the assessment of risk factors for intracerebral hemorrhage in chronic diabetes.

This study was supported by the Ministry of Health of Ukraine, order No. 509 dated 24.02.2020 "Study of endothelium-platelet relations in mechanisms of organoprotective action of drugs under conditions of hyperglycemia and insulin resistance" (0120U101502) and Ministry of Education and Science of Ukraine "Development of biochemical screening test system for determining the state of the blood-brain barrier" (0120U102241).

\section{References}

Anderssohn, M., Schwedhelm, E., Lüneburg, N., Vasan, R. S., \& Böger, R. H. (2010). Asymmetric dimethylarginine as a mediator of vascular dysfunction and a marker of cardiovascular disease and mortality: An intriguing interaction with diabetes mellitus. Diabetes and Vascular Disease Research, 7(2), 105-118.

Avci, E., Karabulut, A., Alp, A. G., Baba, B., \& Bilgi, C. (2020). Crucial markers showing the risk of coronary artery disease in obesity: ADMA and neopterin. Journal of Medical Biochemistry, 39(4), 452-459.

Bo, M. S., Cheah, W. L., Lwin, S., Moe Nwe, T., Win, T. T., \& Aung, M. (2018). Understanding the relationship between atherogenic index of plasma and cardiovascular disease risk factors among staff of an University in Malaysia. Journal of Nutrition and Metabolism, 2018, 7027624.

Butko, Y., Tkacheva, O., \& Gorbach, T. (2013). Oxidizing modification of proteins in case of a burn injury in rats against the topical treatment with new wound healing preparations. Current Issues in Pharmacy and Medical Sciences, 26(4), $372-375$.

Carrizzo, A., Izzo, C., Oliveti, M., Alfano, A., Virtuoso, N., Capunzo, M., Di Pietro, P., Calabrese, M., De Simone, E., Sciarretta, S., Frati, G., Migliarino, S., Dama- to, A., Ambrosio, M., De Caro, F., \& Vecchione, C. (2018). The main determinants of diabetes mellitus vascular complications: Endothelial dysfunction and platelet hyperaggregation. International Journal of Molecular Sciences, 19(10), 2968.

Chen, J., Xu, X-M., Xu, Z., \& Zhang, J. (2019). Animal models of acute neurological injuries. Second ed. Springer International Publishing. Springer Nature Switzerland AG.

Çoban, E. K. (2018). Can TG/HDL Ratio be an accurate predictor in the determination of the risk of cerebrovascular events in youngsters? Sisli Etfal Hastanesi Tip Bulteni, 52(3), 201-205.

De Oliveira, C. O., Reimer, A. G., Da Rocha, A. B., Grivicich, I., Schneider, R. F., Roisenberg, I., Regner, A., \& Simon, D. (2007). Plasma von Willebrand factor levels correlate with clinical outcome of severe traumatic brain injury. Joumal of Neurotrauma, 24(8), 1331-1338.

El Assar, M., Angulo, J., Santos-Ruiz, M., Ruiz de Adana, J. C., Pindado, M. L., Sánchez-Ferrer, A., Hernández, A., \& Rodríguez-Mañas, L. (2016). Asymmetric dimethylarginine (ADMA) elevation and arginase up-regulation contribute to endothelial dysfunction related to insulin resistance in rats and morbidly obese humans. The Journal of Physiology, 594(11), 3045-3060.

Elias, A. N., \& Eng, S. (2005). Homocysteine concentrations in patients with diabetes mellitus-relationship to microvascular and macrovascular disease. Diabetes, Obesity and Metabolism, 7(2), 117-121.

Esse, R., Barroso, M., Tavares de Almeida, I., \& Castro, R. (2019). The contribution of homocysteine metabolism disruption to endothelial dysfunction: State-of-theart. International Joumal of Molecular Sciences, 20(4), 867.

Frohlich, J., \& Dobiásová, M. (2003). Fractional esterification rate of cholesterol and ratio of triglycerides to HDL-cholesterol are powerful predictors of positive findings on coronary angiography. Clinical Chemistry, 49(11), 1873-1880.

Fu, Y., Wang, X., \& Kong, W. (2018). Hyperhomocysteinaemia and vascular injury: Advances in mechanisms and drug targets. British Joumal of Pharmacology, 175(8), 1173-1189.

Gabbay, K. H., Sosenko, J. M., Banuchi, G. A., Mininsohn, M. J., \& Flückiger, R. (1979). Glycosylated hemoglobins: Increased glycosylation of hemoglobin A in diabetic patients. Diabetes, 28(4), 337-340.

Ghasemi, A., Khalifi, S., \& Jedi, S. (2014). Streptozotocin-nicotinamide-induced rat model of type 2 diabetes (review). Acta Physiologica Hungarica, 101(4), 408-420.

Godoy, D. A., Piñero, G. R., Svampa, S., Papa, F., \& Di Napoli, M. (2008). Hyperglycemia and short-term outcome in patients with spontaneous intracerebral hemorrhage. Neurocritical Care, 9(2), 217-229.

Gragnano, F., Sperlongano, S., Golia, E., Natale, F., Bianchi, R., Crisci, M., Fimiani, F., Pariggiano, I., Diana, V., Carbone, A., Cesaro, A., Concilio, C., Limongelli, G., Russo, M., \& Calabrò, P. (2017). The role of von Willebrand factor in vascular inflammation: From pathogenesis to targeted therapy. Mediators of Inflammation, 2017, 5620314 .

Hill, M. D. (2014). Stroke and diabetes mellitus. Handbook of Clinical Neurology, $126,167-174$.

Ho, C. I., Chen, J. Y., Chen, S. Y., Tsai, Y. W., Weng, Y. M., Tsao, Y. C., \& Li, W. C. (2015). Relationship between TG/HDL-C ratio and metabolic syndrome risk factors with chronic kidney disease in healthy adult population. Clinical $\mathrm{Nu}$ trition, 34(5), $874-880$.

Hubbard, W. B., Dong, J. F., Cruz, M. A., \& Rumbaut, R. E. (2021). Links between thrombosis and inflammation in traumatic brain injury. Thrombosis Research, 198, 62-71.

International Diabetes Federation (2019). IDF Diabetes Atlas, 9th edn. International Diabetes Federation.

Kalani, M. (2008). The importance of endothelin-1 for microvascular dysfunction in diabetes. Vascular Health and Risk Management, 4(5), 1061-1068.

Kaur, R., Kaur, M., \& Singh, J. (2018). Endothelial dysfunction and platelet hyperactivity in type 2 diabetes mellitus: Molecular insights and therapeutic strategies. Cardiovascular Diabetology, 17(1), 121.

Lancellotti, P., Ancion, A., D’Orio, V., Gach, O., Maréchal, P., \& Krzesinski, J. M. (2018). Bradykinine et protection cardiovasculaire. Rôle du périndopril, un inhibiteur de l'enzyme de conversion de l'angiotensine [Bradykinin and cardiovascular protection. Role of perindopril, an inhibitor of angiotensin conversion enzyme]. Revue Medicale de Liege, 73(4), 197-205 (in French).

Lau, L. H., Lew, J., Borschmann, K., Thijs, V., \& Ekinci, E. I. (2019). Prevalence of diabetes and its effects on stroke outcomes: A meta-analysis and literature review. Journal of Diabetes Investigation, 10(3), 780-792.

Lee, W., Lee, H. J., Jang, H. B., Kim, H. J., Ban, H. J., Kim, K. Y., Nam, M. S., Choi, J. S., Lee, K. T., Cho, S. B., Park, S. I., \& Lee, H. J. (2018). Asymmetric dimethylarginine (ADMA) is identified as a potential biomarker of insulin resistance in skeletal muscle. Scientific Reports, 8(1), 2133.

Leiper, J., \& Vallance, P. (1999). Biological significance of endogenous methylarginines that inhibit nitric oxide synthases. Cardiovascular Research, 43(3), $542-548$.

Li, Y. W., Kao, T. W., Chang, P. K., Chen, W. L., \& Wu, L. W. (2021). Atherogenic index of plasma as predictors for metabolic syndrome, hypertension and di- 
abetes mellitus in Taiwan citizens: A 9-year longitudinal study. Scientific Reports, 11(1), 9900.

Ma, Y., Li, Z., Chen, L., \& Li, X. (2016). Blood lipid levels, statin therapy and the risk of intracerebral hemorrhage. Lipids in Hhealth and Disease, 15, 43.

Mashayekhi-Sardoo, H., Atkin, S. L., Montecucco, F., \& Sahebkar, A. (2021). Potential alteration of statin-related pharmacological features in diabetes mellitus. BioMed Research International, 2021, 6698743.

Menini, S., Iacobini, C., Vitale, M., Pesce, C., \& Pugliese, G. (2021). Diabetes and pancreatic cancer - a dangerous liaison relying on carbonyl stress. Cancers, 13(2), 313 .

Münch, G., Keis, R., Wessels, A., Riederer, P., Bahner, U., Heidland, A., Niwa, T., Lemke, H. D., \& Schinzel, R. (1997). Determination of advanced glycation end products in serum by fluorescence spectroscopy and competitive ELISA. European Journal of Clinical Chemistry and Clinical Biochemistry, 35(9), 669-677.

Nafisa, A., Gray, S. G., Cao, Y., Wang, T., Xu, S., Wattoo, F. H., Barras, M., Cohen, N., Kamato, D., \& Little, P. J. (2018). Endothelial function and dysfunction: Impact of metformin. Pharmacology and Therapeutics, 192, 150-162.

Nair, D., Nedungadi, D., Mishra, N., Nair, B. G., \& Nair, S. S. (2021). Identification of carbonylated proteins from monocytic cells under diabetes-induced stress conditions. Biomedical Chromatography, 35(6), e5065.

Odegaard, A. O., Jacobs, D. R., Jr., Sanchez, O. A., Goff, D. C., Jr., Reiner, A. P., \& Gross, M. D. (2016). Oxidative stress, inflammation, endothelial dysfunction and incidence of type 2 diabetes. Cardiovascular Diabetology, 15,51 .

Padilla, J., Carpenter, A. J., Das, N. A., Kandikattu, H. K., López-Ongil, S., MartinezLemus, L. A., Siebenlist, U., DeMarco, V. G., \& Chandrasekar, B. (2018). TRAF3IP2 mediates high glucose-induced endothelin-1 production as well as endothelin-1-induced inflammation in endothelial cells. American Journal of Physiology. Heart and Circulatory Physiology, 314(1), H52-H64.

Ren, X., Ren, L., Wei, Q., Shao, H., Chen, L., \& Liu, N. (2017). Advanced glycation end-products decreases expression of endothelial nitric oxide synthase through oxidative stress in human coronary artery endothelial cells. Cardiovascular Diabetology, 16(1), 52 .

Saliba, W., Barnett-Griness, O., Gronich, N., Molad, J., Naftali, J., Rennert, G., \& Auriel, E. (2019). Association of diabetes and glycated hemoglobin with the risk of intracerebral hemorrhage: A population-based cohort study. Diabetes Care, $42(4), 682-688$.
Shahidi, M. (2017). Thrombosis and von Willebrand factor. Advances in Experimental Medicine and Biology, 906, 285-306.

Shi, Y., \& Vanhoutte, P. M. (2017). Macro- and microvascular endothelial dysfunction in diabetes. Journal of Diabetes, 9(5), 434449.

Škovierová, H., Vidomanová, E., Mahmood, S., Sopková, J., Drgová, A., Červeňová, T., Halašová, E., \& Lehotský, J. (2016). The molecular and cellular effect of homocysteine metabolism imbalance on human health. International Joumal of Molecular Sciences, 17(10), 1733.

Snarska, K. K., Bachórzewska-Gajewska, H., Kapica-Topczewska, K., Drozdowski, W., Choraż̇y, M., Kułakowska, A., \& Małyszko, J. (2017). Hyperglycemia and diabetes have different impacts on outcome of ischemic and hemorrhagic stroke. Archives of Medical Science, 13(1), 100-108.

Strain, W. D., \& Paldánius, P. M. (2021). Correction to: Diabetes, cardiovascular disease and the microcirculation. Cardiovascular Diabetology, 20(1), 120.

$\mathrm{Su}$, J. B. (2015). Vascular endothelial dysfunction and pharmacological treatment. World Journal of Cardiology, 7(11), 719-741.

Wang, X., Shi, N., Shi, H., Ye, H., Li, N., Sun, P., Bai, D., \& Yuan, H. (2018). Correlations of acute cerebral hemorrhage complicated with stress ulcer bleeding with acute physiology and chronic health evaluation (APACHE) II score, endothelin (ET), tumor necrosis factor-alpha (TNF- $\alpha$ ), and blood lipids. Medical Science Monitor, 24, 9120-9126.

Yaribeygi, H., Sathyapalan, T., Atkin, S. L., \& Sahebkar, A. (2020). Molecular mechanisms linking oxidative stress and diabetes mellitus. Oxidative Medicine and Cellular Longevity, 2020, 8609213.

Yokota H. (2007). Cerebral endothelial damage after severe head injury. Journal of Nippon Medical School = Nippon Ika Daigaku zasshi, 74(5), 332-337.

Yu, Z., Tang, L., Chen, L., Li, J., Wu, W., \& Hu, C. (2015). Role for HIF-1 $\alpha$ and downstream pathways in regulating neuronal injury after intracerebral hemorrhage in diabetes. Cellular Physiology and Biochemistry: International Journal of Experimental Cellular Physiology, Biochemistry, and Pharmacology, 37(1), 67-76.

Zambon A. (2020). Non- high-density lipoprotein cholesterol and cardiovascular disease in patients with diabetic dyslipidaemia. Diabetes Mellitus, 23(1),65-71.

Zhu, X., Yu, L., Zhou, H., Ma, Q., Zhou, X., Lei, T., Hu, J., Xu, W., Yi, N., \& Lei, S. (2018). Atherogenic index of plasma is a novel and better biomarker associated with obesity: a population-based cross-sectional study in China. Lipids in Health and Disease, 17(1), 37. 